\title{
Apoyo a la autonomía en Educación Física: antecedentes, diseño, metodología y análisis de la relación con la motivación en estudiantes adolescentes Autonomy support Physical Education: history, design, methodology and analysis regarding motivation in teenage students
}

\author{
Antonio Baena-Extremera*, Antonio Granero-Gallegos **, JoséAntonio Sánchez-Fuentes*, Marina Martínez-Molina* \\ *Universidad de Murcia, ** Centro del profesororado Cuevas-Olula (Cuevas del Almanzora)
}

Resumen: En cualquier área de la enseñanza es reconocida la importancia que tiene que los alumnos están motivados. Pero para ello hacen falta profesores que motiven y actuaciones docentes que provoquen este estado en los estudiantes. El apoyo a la autonomía puede ser clave para mejorar la motivación en los discentes, así como un indicador para buscar otras mejoras en el proceso de enseñanza-aprendizaje. El objetivo de este trabajo ha sido analizar la importancia que puede tener el apoyo a la autonomía en el alumno (tanto en su aprendizaje como en la adquisición de hábitos) y ejemplificar el diseño, metodología y posibles análisis a realizar para responder a los objetivos planteados. Para ello se utilizará una muestra de 758 alumnos (347 hombres, 45.8\%; 411 mujeres, 54.2\%) de secundaria de la Región de Murcia, con edades comprendidas entre 12 y 18 años $(M=15.22 ; S D=1.27)$. El instrumento a utilizar será un cuestionario compuesto por las escalas: Learning Climate Quetionarire (LCQ), Sport Motivation Scale (SMS), Intention to partake in leisure-time physical activity (Intención-PFTL), Escala de Satisfacción Intrínseca hacia la Educación Física (SSI-EF) y la escala de Importancia y utilidad de la Educación Física (IEF). Los posibles resultados podrán mejorar y discutir muchos de los trabajos ya existentes y aportar nuevas orientaciones que sirvan al profesorado para mejorar su actuación docente.

Palabras clave: apoyo a la autonomía; motivación; educación física.

Abstract: In any area of education it is recognized how important is that students are motivated. But this requires teachers who motivate and actions that cause this state on students. The autonomy support may be the key to improve the motivation of learners, as well as an indicator to search for other improvements in the teaching-learning process. The aim of this study was to analyze the potential importance of supporting autonomy in students (both in learning and in the acquisition of habits) and exemplify the design, methodology and analysis to make possible to get the objectives. This will draw a sample of 758 high school students (347 men, 45.8\%; 411 women, 54.2\%) of the Region of Murcia, aged between 12 and 18 ( $M=15.22$, SD = 1.27). The instrument to be used is a questionnaire consisting of scales: Learning Climate Quetionarire (LCQ), Sport Motivation Scale (SMS), Intention to partake in leisure-time physical activity (Intention-PFTL), Sport Satisfaction Instrument to Physical Education (SSI-EF) and the scale of Importance and usefulness of Physical Education (IEF). Possible results may improve and discuss many of the existing work and provide further guidance to be used for teachers to improve their teaching performance.

Key words: autonomy support; motivation; physical education.

\section{Introducción}

Numerosas investigaciones (Ryan \& Deci, 2007; Ryan, FrederickRecascino, Lepes, Rubio \& Sheldon, 1997; Ryan, William, Patrick \& Deci, 2009), están demostrando los beneficios que supone tener alumnado con cierta motivación hacia las asignaturas y los contenidos que aprenden. De hecho, independientemente del área o etapa educativa en la que se encuentren, los estudiantes necesitan estar motivados para participar de manera activa en el proceso de enseñanza-aprendizaje, de forma que se asienten las bases en ellos con tal fuerza que continúen aprendiendo durante toda su vida (Boekaerts, 1996; Phalet \& Lens, 1995). Por ello, en los últimos años la motivación está siendo estudiada desde diversas teorías, como la Teoría de laAutodeterminación(Deci \& Ryan, 1985; 2000) o la Teoría de Metas de Logro (Nicholls, 1984), para intentar descifrar algunos de los condicionantes que puedan influir en la educación de los discentes.

Entre esos condicionantes cabe destacar uno que está obteniendo una gran importancia actualmente, como es la autonomía del estudiante. Hablamos de autonomía cuando el profesor delega en sus pupilos responsabilidad para actuar de forma voluntariosa en vistas de su propio aprendizaje o, como definen Williams, Saizow, Ross y Deci (1997), se entendería como el grado en el que los profesores reconocen la capacidad de los alumnos y fomentan su participación activa en actividades de aprendizaje. Estaríamos hablando pues, de un proceso activo por parte de los docentes que según estos autores facilitaría la participación de los estudiantes en su aprendizaje autodeterminado.

Diversas investigaciones (Black \& Deci, 2000; Deci, Schwartz, Sheinman \& Ryan, 1981; Reeve, 2002) han hecho hincapié en que

Fecha recepción: 04-03-13- Fecha envío revisores: 04-03-13- Fecha de aceptación: 21-04-13 Correspondencia: Antonio Baena Extremera

C/ Argentina s/n 30720

Santiago de la Ribera (Murcia)

Email: abaenaextrem@um.es cuando en clase se crean climas de aprendizaje que apoyan la autonomía de los estudiantes, éstos mejorarán su motivación intrínseca; mientras que aquellos en los que se percibe un mayor control de su comportamiento por parte del profesor, provocarán una disminución de su sentido de la voluntad y, consecuentemente, una reducción de la motivación natural. De hecho, Matos (2009) entiende que la motivación intrínseca es el mejor ejemplo del comportamiento autónomo y, por tanto, autodeterminado; mientras que la motivación extrínseca supondría un mayor control en el alumno y, evidentemente, sería menos autónoma.

Por consiguiente, sería muy interesante estudiar inicialmente los factores que puedan influir en conseguir la autonomía adecuada en los alumnos, dependiendo posiblemente, de factores disposicionales y ambientales, y como ésta autonomía puede influir en la motivación de nuestros estudiantes.

En el estudio de la autonomía del alumno, Williams y Deci (1996) elaboraron el Learning Climate Questionnaire (LCQ), un cuestionario compuesto por 15 ítems agrupados en un único factor. De éste existe también una versión reducida de 6ítems. Ésta versiónúltima fue utilizada en los trabajos de Hagger, Chatzisarantis, Culverhouse y Biddle(2003), Standage, Duda y Ntoumanis (2005), Ntoumanis (2005), Standage, Gillison, Ntoumanis y Treasure (2012), con estudiantes de Educación Física (EF) en secundaria.

En España esta escala fue adaptada inicialmente al deporte por Balaguer, Castillo, Duda y Tomás (2009), dando lugar al Cuestionario de Clima en el Deporte, pero en cambio, no existe aún trabajos llevados a cabo en el campo de la Educación Física (EF). Exclusivamente, se encuentran trabajos con este instrumento fuera de España, como los ya nombrados o el de Hagger et al. (2003) y Ntoumanis (2005).

Por todo ello, el trabajo que aquí se presenta es de relevancia, pues la validación a la EF de dicha escala y su utilización de forma conjunta con otras ya existentes, puede llegar a aportar información de tanto valor, que podría contribuir a mejorar algunos de los problemas con los que hoy día se enfrenta el profesorado, incluidos algunos problemas de conducta de los estudiantes. Asimismo, teniendo en cuanta la urgencia 
que se está manifestando en los últimos tiempos por reducir la inactividad escolar y la necesidad de que los adolescentes realicen actividad física, resulta determinante conocer qué aspectos pueden mejorar la autonomía de los jóvenes y la relación e influencia en la motivación, satisfacción/ diversión e importancia y utilidad de la EF por los alumnos, con vista a incrementar las prácticas escolares de actividad física. Por tanto, el planteamiento que se persigue con todo este trabajo estaría reflejado en los siguientes objetivos.

\section{Metodo}

\section{Objetivos del estudio}

A continuación se describen los objetivos fundamentales del trabajo que se pretende desarrollar:

1.- Validar al castellano, y adaptada a la EF, la escala «Learning Climate Questionaire» (LCQ) para evaluar la percepción de apoyo a la autonomía por parte del alumnado.

2.-Analizar la influencia del apoyo a la autonomía sobre motivación autodeterminada. Para ello, y de forma preliminar, se pretende aportar evidencias sobre la dimensionalidad de la versión española del Sport Motivation Scale adaptado a la EF en una muestra de adolescentes estudiantes de educación secundaria mediante procedimientos confirmatorios.

3.- Conocer las relaciones de predicción entre el apoyo a la autonomía, la motivación autodeterminada y la intención de práctica de actividad físico-deportiva.

4.- Conocer las relaciones de predicción entre el apoyo a la autonomía, la motivación, la satisfacción intrínseca y la importancia de laEducación Física.

5.-Analizar los perfiles motivacionales del alumnado de educación secundaria en EF y la inclusión en los mismos del apoyo a la autonomía, satisfacción, importancia y utilidad a la EF, así como la intención de práctica futura de actividad física.

\section{Muestra}

En este estudio ya se ha llevado acabo el trabajo de campo, y en el mismo participaron un total de 758 alumnos ( 347 hombres $=45.8 \%$; 411 mujeres $=54.2 \%$ ) de secundaria de la Región de Murcia. El rango de edad estuvo comprendido entre 12 y 18 años $(M=15.22$; $S D=1.27)$, siendo la edad media de los chicos $15.2(S D=1.29$ y la de las chicas $15.18(S D=1.26)$. La distribución por cursos fue la siguiente: 343 (45.3\%) estudian $2^{\circ}$ de ESO; 152 (20.1\%), $3^{\circ}$ de ESO; 206 (27.2\%), $4^{\circ}$ de ESO; y 57 (7.5\%), $1^{\circ}$ de bachillerato.

\section{Instrumentos}

Para llevar a cabo esta investigación, se elaboró un cuestionario compuesto de las siguientes escalas:

Learning Climate Quetionarire (LCQ). Se adaptóla versión original del Learning Climate Quetionarire (LCQ) de Wiliamns y Deci (1996), basada en el Health-Care Climate Questionnaire (Williams, Grow, Freedman, Ryan \& Deci, 1996). Este instrumento en su versión original consta de 15 ítems para medir el apoyo a la autonomía por parte del profesor mediante una única dimensión: el apoyo a la autonomía. En las instrucciones que se les da a los alumnos para rellenar este cuestionario se les pide que indiquen el grado de acuerdo con los ítems, recogiéndose las respuestas en una escala de ítems politómicos de 7 puntos que oscila desde Muy en desacuerdo (1) a Muy de acuerdo (7).

Sport Motivation Scale (SMS). Se empleó la versión validada al castellano por Núñez, Martín-Albo, Navarro y González (2006) y adaptada a la EF. La escala original se denominó Échelle de Motivation dans les Sports (ÉMS; Brière, Vallerand, Blais \& Pelletier, 1995) y fue traducida al inglés por Pelletier et al., (1995) pasando a denominarse Sport Motivation Scale (SMS). Consta de 28 ítems que miden los diferentes tipos de motivación establecidos por la Teoría de la Autodeterminación y que sugiere la explicación multidimensional de la motivación: desmotivación (4 ítems), motivación extrínseca (ME) (12 ítems; regulación externa, introyectada e identificada; 4 ítems para cada subescala), y motivación intrínseca (MI) (12 ítems). Las respuestas se recogieron en una escala de ítems politómicos con rango de puntuación entre 1 (Totalmente en desacuerdo) y 7 (Totalmente de acuerdo).

Intention to partake in leisure-time physical activity (IntenciónPFTL). Se utilizó la versión española adaptada a EF (Granero-Gallegos, Baena-Extremera, Pérez-Quero \& Bracho-Amador, 2013) del original de Chatzisarantis, Biddle y Meek (1997). Esta escala consta de tres ítems redactados sobre la base de la obra de Ajzen y Madden (1986), teniendo en cuenta que la redacción correspondiera con el criterio de comportamiento en el tiempo, el contexto, objetivo y acción (Ajzen \& Fishbein, 1980). El instrumento trata de evaluar la intención de los estudiantes de ser físicamente activos en su tiempo libre durante el período de un mes. Para ello respondieron a tres preguntas, sobre el encabezado de «En mi tiempo libre, fuera del instituto...»: (1) Tengo intención de hacer ejercicio físico al menos tres veces por semana en el próximo mes; (2) Tengo planeado hacer ejercicio físico al menos tres veces por semana en el próximo mes; (3) Estoy decidido a hacer ejercicio físico al menos tres veces por semana en el próximo mes. Las respuestas se recogen en una escala de ítems politómicos de 7 puntos que oscilan entre 1 (muy improbable) y 7 (muy probable).

Sport Satisfaction Instrument (SSI). Se utilizó la versión española del Sport Satisfaction Instrument adaptada a EF (SSI-EF) (BaenaExtremera, Granero-Gallegos, Bracho-Amador \& Pérez-Quero, 2012) del original Sport Satisfaction Instrument (Balaguer, Atienza, Castillo, Moreno \& Duda, 1997; Duda \& Nicholls, 1992). El SSI-EF consta de 8 ítems para medir la satisfacción intrínseca en una actividad deportiva mediante dos subescalas que miden satisfacción/diversión (5 ítems) y aburrimiento (3 ítems) en la práctica deportiva. En las instrucciones se pide a los sujetos que indiquen su grado de acuerdo con los ítems que reflejan criterios de diversión o aburrimiento, recogiéndose las respuestas en una escala de ítems politómicos de 5 puntos que oscila desde muyen desacuerdo (1) a muy de acuerdo (5).

Importancia y utilidad de la Educación Física (IEF). Se midió la importancia y utilidad concedida por el alumnado a la EF (Moreno, González-Cutre \& Ruiz, 2009) a través de 3 ítems. Los alumnos debían responder en una escala deítems politómicos con un rango de puntuación entre 1 (totalmente en desacuerdo) y 4 (totalmente de acuerdo).

Asimismo, se utilizaron otras variables de identificación para comprobar la interacción de las mismas con los perfiles hallados y las subdimensiones estudiadas: sexo (varones; mujeres), práctica deactividad física fuera del centro educativo (sí; no) y, en caso afirmativo, $n^{\circ}$ de horas semanales que dedica a la práctica de ejercicio físico (1 hora o menos; de 2 a 4 horas; más de 3 horas).

\section{Descripción de algunos de los modelos planteados}

Las posibilidades de investigación con estos instrumentos podría ser enorme pero, obviamente, es necesario acotar y evaluar diferentes diseños de modelos de ecuaciones estructurales, pero partiendo de una idea general (ver figura 1). Agrandes rasgos, desdeel apoyo a la autonomía se intentaría predecir la motivación del alumnado y como ésta predice el resto de variables objeto de estudio. Al final, se intentará dar respuesta a la influencia del apoyo a la autonomía, a través de la motivación autodeterminada, en la importancia y utilidad de la EF y, sobre todo, en la intención de práctica futura de actividad física en tiempo libre.

Tal como se ha comentado la intención es testear diferentes modelos a partir de esta estructura, hasta conseguir modelos con buenos indicadores de ajuste.

\section{Análisis estadístico}

Para llevar a cabo este trabajo, el planteamiento de análisis estadístico debe pasar por lo siguiente:

En relación al primer objetivo planteado, y que hace referencia a la validación al castellano de la escala $L C Q$ en su versión original y su versión reducida, se partirá de un estudio preliminar realizado con un total de 300 alumnos de educación secundaria y características similares a los que participaron en el trabajo de campo de esta investigación que se presenta. En esta adaptación se siguieron las directrices propuestas 


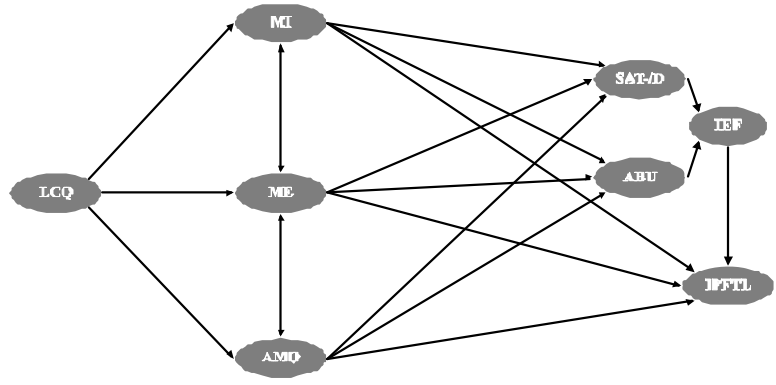

Figura 1. Posibles modelos a testear. Abreviaturas de las diferentes subescalas: AUT = apoyo a la autonomía; $\mathrm{MI}=$ m $\mathrm{SAT} / \mathrm{D}$ = satisfacción / diversión; $\mathrm{ABU}$ = aburrimiento; IEF = importancia y utilidad de la EF IPFTL = intención de práctica física en tiempo libre.

por Carretero-Dios y Pérez (2007) para estudios instrumentales.

En ese análisis preliminar se realizó la traducción y fases previas del proceso de adaptación del instrumento al español. Esta adaptación española de la escala se realizó atendiendo a los estándares metodológicos internacionales recomendados por la International Test Comision(ITC) para adaptar correctamente test y escalas de unas culturas a otras (Hambleton, 2005; Muñiz \& Bartram, 2007; Muñiz, 2000). Para evitar imprecisiones, se combinaron los diseños de traducción directa e inversa de los ítems, según el procedimiento parallel back translation (Brislin, 1986). La evaluación cualitativa de ítems (validez de contenido) se efectuó mediante el juicio de cuatro expertos (Osterlind, 1989): dos en construcción de escalas y dos conocedores del constructo a evaluar. Todos los ítems fueron analizados y revisados se llegó a hasta que se recogiese la dimensión teórica de la forma más clara y precisa. A continuación se realizó un análisis deítems y homogeneidad de la escala, así como un análisis factorial exploratorio (AFE) para obtener la estructura factorial interna y analizar la consistencia interna con el objeto de determinar la fiabilidad (alfa) de Cronbach.

A partir de estos datos, y con la muestra de 758 alumnos se examinará su estructura factorial con análisis factorial confirmatorio (AFC) con el programa LISREL; se evaluarála consistencia interna mediante alfa de Cronbach, coeficiente de fiabilidad compuesta y varianza media extractada (AVE); se verificará su estabilidad temporal; y se evaluará la validez convergente del instrumento, analizando su relación con los distintos tipos de motivación autodeterminada mediante Modelos de Ecuaciones Estructurales. De esta manera se llegaráa la versión definitiva del $L C Q$ adaptado a la EF.

Para el segundo objetivo se analizarán las propiedades psicométricas de los tres modelos hipotetizados del SMS (tres, cinco y siete factores) y adaptado a la EF. Para ello se realizaran los siguientes procedimientos de análisis: examen de la estructura factorial de cada modelo con AFC con el programa LISREL; evaluación de la consistencia interna mediante alfa de Cronbach, coeficiente de fiabilidad compuesta y AVE; determinación del modelo que se ajusta mejor a los datos -mediante análisis de las diferencias entre los valores de $X^{2}$ asociados con los modelos anidados $\left(\ddot{\mathrm{A}} X^{2}\right)$ y cálculo del índice de validación cruzada esperada (ECVI) de cada modelo, que contempla tanto el ajuste como la parsimonia del modelo, ajustando mejor el que presente un valor más bajo-; y evaluación de la validez convergente.

En el caso del objetivo número 3 y 4, se llevarán a cabo análisis estadísticos descriptivos y de correlaciones (coeficiente de Pearson) entre las dimensiones a analizar: (a) en el objetivo 3: $L C Q-E F$, IntenciónPFTL y subescalas del SMS; (b) en el objetivo 4: $L C Q-E F$, subescalas del SMS, subescalas del SSI-EF e IEF. Para analizar las influencias y relaciones de predicción entre factores se analizarán Modelos de Ecuaciones Estructurales con el programa LISREL.

Para el quinto objetivo se parte de la siguiente hipótesis: se cree que los perfiles motivacionales más autodeterminados incluirán el apoyo a la autonomía, los de satisfacción, así como los mayores índices de importancia y utilidad de la EF y de intención de práctica futura.
En este caso se realizará un análisis de cluster con el programa SPSS. Esta técnica multivariante busca agrupar elementos (o variables) para lograr la máxima homogeneidad en cada grupo y las mayores diferencias entre ellos. Lo ideal es llevar a cabo dos análisis de conglomerados, atendiendo las sugerencias de Hair, Black, Babin y Anderson (1999). Primero se divide la muestra de forma aleatoria en dos grupos y se realiza un análisis de cluster jerárquico exploratorio con el método de Ward (grupo 1) y en base a la lectura dendograma y a los resultados lógicos se selecciona una solución. Seguidamente se verifican estos resultados obteniendo los perfiles motivacionales del grupo 2 mediante análisis de cluster a través del método $k$-medias. Finalmente, se realiza un último análisis de cluster con toda la muestra a través del método de k-medias. A continuación se examinarán las diferencias entre los perfiles y los diferentes grupos en los que el sexo, la práctica de actividad física en tiempo de ocio y el número de horas de práctica físico-deportiva semanal actuarán como variables independientes.

\section{Resultados y discusión}

El llevar a cabo un trabajo de esta envergadura supone obtener resultados de una importancia tal, que pueda hacer cuestionarse muchas de las situaciones cotidianas de enseñanza. De hecho, con los resultados se espera demostrar nuevas formas de planificar y organizar la enseñanza en EF, nuevas formas de trabajar y, sobre todo, descubrir el modo de potenciar la importancia del alumno hacia la EF y la intención de práctica de actividad física en su tiempo libre.

El validar al español la escala de apoyo a la autonomía en alumnos de educación secundaria puede servir para obtener información que ayude al profesorado no solo a organizar su tarea docente, sino a diseñar programas que mejoren ésta variable en busca de otras consecuencias positivas en los alumnos. Por ejemplo, el apoyo a la autonomía promueve la motivación, y la motivación autodeterminada se ha demostrado en diversas investigaciones estar vinculada con una mayor concentración en la clase (Black \& Deci, 2000; Standage, Duda \& Ntoumanis, 2005), un mayor esfuerzo académico (Ntoumanis, 2001) y un menor abandono escolar (Black \& Deci, 2000), entre otros beneficios. Igualmente, el apoyo a la autonomía por parte del profesorado de EF es un predictor preferentemente de las formas más autodeterminadas de motivación entre los estudiantes adolescentes, coincidiendo con las aportaciones de Black y Deci (2000), Hagger et al. (2003, 2009), Taylor y Ntoumanis (2007) y Standage et al. (2012). En sentido contrario, cuando el clima de clase en EF no apoya la autonomía, los niveles de motivación intrínseca bajan en los alumnos (Vallerand, 2000). Por ello, es de destacar la importancia que tiene esta escala y su gran utilidad para los docentes, tanto en la creación de clima de aprendizaje como en la creación de climas motivacionales, y la repercusión de éstos en los alumnos.

En relación a las posibles predicciones a través de los modelos de ecuaciones estructurales, hay que tener en cuenta, tal como muestran diversas investigaciones, que la motivación autodeterminada se relaciona positivamente con un mayor compromiso, adherencia a la práctica deportiva, esfuerzo y persistencia (Escartí \& Gutiérrez, 2001; FerrerCaja \& Weiss, 2000; Hein, Muur \& Koka, 2004; Moreno, Cervelló \& González-Cutre, 2007; Sproule, Wang, Morgan, McNeill \& McMorris, 2007; Standage, Duda \& Ntoumanis, 2003). Por ello, es de destacar que los resultados que aquí se puedan encontrar son especialmente importantes en la promoción de un estilo de vida activo entre los adolescentes y que la asignatura de EF tendría un papel activo ante esta situación. Además, habría que tener presente la influencia que éstos resultados puedan tener en la mejora de la salud pública (Sallis \& Mckenzie, 1991) de cada país.

Además, por un lado, se ha de tener en cuenta que, según exponen Hagger et al. (2003), el apoyo a la autonomía influye en la intención de práctica de ejercicio físico en tiempo libre; y, por otro lado, que la importancia concedida a la EF entre los estudiantes adolescentes se relacionan con perfiles autodeterminados o de alta motivación, así como con un número mayor de horas de práctica deportiva en tiempo de ocio 
(Granero-Gallegos, Baena-Extremera, Pérez-Quero \& Bracho-Amador, 2012; Moreno-Murcia, Zomeño, Marín, Ruiz \& Cervelló, en prensa). Así pues, la asignatura de EF y el profesorado que se encarga de la misma tienen un papel decisivo en esta intervención. Los datos del presente estudio serían en este caso, muy importantes de cara a la preparación del profesorado y la organización de la docencia a nivel metodológico

\section{Referencias}

Ajzen, I., \& Fishbein, M. (1980). Understanding Attitudes and Predicting Social Behaviour. Englewood-Cliffs, NJ: Prentice-Hall.

Ajzen, I., \& Madden, T. (1986). Prediction of goal-directed behaviour:Attitudes, intentions and perceived behavioural control. Journal of Experimental Social Psychology, 22, 453-474.

Baena-Extremera, A., Granero-Gallegos,A., Bracho-Amador, C., \& Pérez-Quero, F. J. (2012). Versión española del Sport Satisfaction Instrument (SSI) adaptado a la Educación Física. Revista de Psicodidáctica, 17(2), 377-395. Recuperado de: http:// www.ehu.es/ojs/index.php/psicodidactica/article/view/4037.DOI:10.1387 Rev.Psicodidact.4037

Balaguer, I., Atienza, F. L., Castillo, I., Moreno, Y., \& Duda, J. L. (1997). Factorial structure of measures of satisfaction/interest in sport and classroom in the case of Spanish adolescents. Abstracts of $4^{\text {thEuropean }}$ Conference of Psychological Assessment (p. 76). Lisbon: Portugal.

Balaguer, I., Castillo, I., Duda, J. L. \& Tomás, I. (2009). Análisis de las propiedades psicométricas de la versión española del cuestionario de clima en el deporte. Revista de Psicología del Deporte, 18(1), 73-83. Recuperado de: http://redalyc.uaemex.mx/ redalyc/pdf/2351/235119250005.pdf

Black, A.E., \& Deci, E. L.(2000). The effects of instructors' autonomy support and students' autonomous motivation on learning organic chemistry: A self-determination theory perspective. Science Education, 84, 740-756.

Boekaerts, M. (1996). Self-regulated learning at the junction of cognition and motivation. European Psychologist, 1(2), 100-112.

Brière, N., Vallerand, R., Blais, N., \& Pelletier, L. (1995). Développement et validation d'une mesure de motivation intrinsèque, extrinsèque et d'amotivation en contexte sportif: l'Échelle de motivation dans les sports (ÉMS). International Journal of Sport Psychology, 26, 465-489.

Brislin, R. W. (1986). The wording and translation of research instruments. In W. Lonnery J. Berry (Eds.), Field methods in cross-cultural research (pp. 137-164). Beverly Hills, CA: Sage.

Carretero-Dios, H., \& Pérez, C. (2007). Standards for the development and the review of instrumental studies: Considerations about test selection in psychological research. International Journal of Clinical and Health Psychology, 7, 863-882. Recuperado de http://www.aepc.es/ijchp/articulos_pdf/ijchp-257.pdf

Chatzisarantis, N. L. D., Biddle, S. J.H., \& Meek, G A. (1997). A self-determination theory approach to the study of intentions and the intention-behaviour relationship in children's physical activity. British Journal of Health Psychology, 2, 343-360.

Deci, E. L., Schwartz, A. J., Sheinman, L., \& Ryan, R. M. (1981). An instrument to asses adults' orientations toward control versus autonomy with children: Reflections on intrinsic motivation and perceived competence. Journal of Educational Psychology, 73, 642-650.

Deci, E. L., \& Ryan, R. M. (1985). Intrinsic motivation and self-determination in human behavior. New York: Plenum.

Deci, E. L., y Ryan, R. M. (2000). The «what» and «why» of goal pursuits: Human needs and the self-determination of behavior. Psychological Inquiry, 11, 227-268.

Duda, J. L., \& Nicholls, J. G. (1992). Dimensions of achievement motivation in schoolwork and sport. Journal of Educational Psychology, 84(3), 290-299.

Escartí, A., \& Gutiérrez, M. (2001). Influence of the motivational climate in physica education on the intention to practice physical activity or sport. European Journal of Sport Science, 1, 1-12.

Ferrer-Caja, E., \& Weis, M. R. (2000). Predictors of intrinsic motivation among adolescen students in physical education. Research Quarterly for Exercise and Sport, 71, 267-279.

Granero-Gallegos, A., Baena-Extremera, A., Pérez-Quero, F. J., \& Bracho-Amador, C. (2012). Analysis of motivational profiles of satisfaction and importance of physical education in high school adolescents. Journal of Sports Science \& Medicine, 11, 614-623.

Granero-Gallegos, A., Baena-Extremera, A., Pérez-Quero, F. J., \& Bracho-Amador, C. (2013). Analysis of intent to partake in leisure-time physical activity teenage students. Paper en evaluación.

Hagger, M. S., Chatzisarantis, N. L., Hein, V., Pihu, M., Soos, I.,... Leemans, S. (2009). Teacher, peer, and parent autonomy support in physical education and leisure-time physical activity: A trans-contextual model of motivation in four nations. Psychology and Health, 24, 689-711.

Hagger, M. S., Chatzisarantis, N., Culverhouse, T., \& Biddle, S. J. H. (2003). The processes by which perceived autonomy support in physical education promotes leisuretime physical activity intentions and behavior: A trans-contextual model. Journal of Educational Psychology, 95, 784-795.

Hair, J. F., Black, W. C., Babin, B. J., \& Anderson, R. E. (1999). Multivariate Data Analysis (7th ed.). New York: Pearson Prentice Hall.

Hambleton, R. K. (2005). Issues, designs and technical guidelines for adapting tests into multiple languages and cultures. En R.K. Hambleton, P.F. Merenday S.D. Spielberger (Eds.), Adapting educational and psychological tests for cross-cultural assessment (pp. 3-38). Mahwah, NJ: Lawrence Erlbaum AssociatesHein, V., Muur,
M., y Koka, A. (2004). Intention to be physically active after school graduation and its relationship to three types of intrinsic motivation. European Physical Education Review, 10, 5-19.

Hein, V., Muur, M., \& Koka, A. (2004). Intention to be physically active after school graduation and its relationship to three types of intrinsic motivation. European Physical Education Review, 10, 5-19.

Matos, L. (2009). Adaptación de dos cuestionarios de motivación: Autorregulación del Aprendizaje y Clima de Aprendizaje. Persona, 12, 167-185. Recuperado de: http:/ redalyc.uaemex.mx/src/inicio/ArtPdfRed.jsp?iCve=147117618010

Moreno, J.A., Cervelló, E. M., \& González-Cutre, D. (2007). Young athletes’ motivational profiles. Journal of Sports Science and Medicine, 6, 172-179.

Moreno, J. A., González-Cutre, D., \& Ruiz, L. M. (2009). Self-determined motivation and physical education importance. Human Movement, 10(1), 5-11.

Moreno-Murcia, J. A., Zomeño, T., Marín, L. M., Ruiz, L. M., \& Cervelló, E. (en prensa). Percepción de la utilidad e importancia de la educación física según la motivación generada por el docente. Revista de Educación.

Muñiz, J. (2000). Adaptación de los test de unas culturas a otras. Metodología de las Ciencias del Comportamiento, 2(2), 129-149.

Muñiz, J., \& Bartram, D. (2007). Improving international tests and testing. European Psychologist, 12, 206-219. DOI:10.1027/1016-9040.12.3.206

Nicholls, J. G. (1984). Achievement motivation: Conceptions of ability, subjective experience, task choice, and performance. Psychological Review, 91, 328-346.

Ntoumanis, N. (2001). A self-determination approach to the understanding of motivation in physical education. British Journal of Educational Psychology, 71(2), 225242

Ntoumanis, N. (2005). A Prospective Study of Participation in Optional School Physical Education Using a Self-Determination Theory Framework. Journal of Educational Psychology, 97, 444-453.

Núñez, J. L., Martín-Albo, J., Navarro, J. G., \& González, V.M. (2006). Preliminary validation of a Spanish version of the Sport Motivation Scale. Perceptual and Motor Skills, 102, 919-930.

Osterlind, S. J. (1989). Constructing Test Items. Londres: Kluwer Academic Publishers.

Phalet, K., \& Lens, W. (1995).Achievement motivation and group loyalty among Turkish and Belgian youngsters. En P.R. Pintrich y M. Maehr (eds.), Advances in motivation and achievement, Vol.9 (pp. 31-72).Greenwich, Conn: JAI Press.

Pelletier, L. G., Fortier, M. S., Vallerand, R. J., Tuson, K. M., Brière, N. M., \& Blais, M. R. (1995). Toward a new measure of intrinsic motivation, extrinsic motivation, and amotivation in sports: the Sport Motivation Scale (SMS). Journal of Sport and Exercise Psychology, 17, 35-53.

Reeve, J. (2002). Self-determination theory applied to educational settings. En E. L. Deci, y R. M. Ryan (Eds.), Handbook of self- determination research. Rochester: The University of Rochester Press.

Ryan, R. M., \& Deci, E. L. (2007). Active human nature: Self-determination theory and the promotion and maintenance of sport, exercise, and health. In M. S. Hagger, y N. L. D. Chatzisarantis (Eds.), Intrinsic motivation and self-determination in exercise and sport (pp. 1-19). Champaign, IL: Human Kinetics.

Ryan, R. M., Frederick-Recascino, C. M., Lepes, D., Rubio, N., y Sheldon, K. M. (1997) Intrinsic motivation and exercise adherence. International Journal of Sport Psychology, 28, 335-354.

Ryan, R. M., Williams, G. C., Patrick, H., \& Deci, E. L. (2009). Self-determination theory and physical activity: The dynamics of motivation in development and wellness. Hellenic Journal of Psychology, 6, 107-124.

Sallis, J. F., \& McKenzie, T. L. (1991). Physical education’s role in public health. Research Quarterly for Exercise and Sport, 62, 124-137.

Standage, M., Duda, J. L., \& Ntoumanis, N. (2003). A model of contextual motivation in physical education: Using constructs from self-determination and achievement goa theories to predict physical activity intentions. Journal of Educational Psychology, 95, 97-110.

Standage, M., Duda, J. L. \& Ntoumanis, N. (2005). A test of self-determination theory in sxhool physical education. British Journal of Educational Psychology, 75(3), 411-433.

Standage, M., Gillison, F. B., Ntoumanis, N., \& Treasure, D. (2012). Predicting Students' Physical Activity and Health-Related Well-Being: A Prospective Cross-Domain Investigation of Motivation Across School Physical Education and Exercise Settings. Journal of Sport \& Exercise Psychology, 34, 37-60. Recuperado de: http://www.selfdeterminationtheory.org/SDT/documents/2012_StandageGillison NtoumanisTreasure JSEP.pdf

Sproule, J., Wang, C. K. J., Morgan, K., McNeill, M., \& McMorris, T. (2007). Effects of motivational climate in Singaporean physical education lessons on intrinsic motivation and physical activity intention. Personality and Individual Differences, 43, 1037-1049.

Taylor, I. M., \& Ntoumanis, N. (2007). Teacher Motivational Strategies and Student SelfDetermination in Physical Education. Journal of Educational Psychology, 99(2), 747-760.

Vallerand, R. J. (2000). Deci and Ryan's self-determination theory: A view from the hierarchical model of intrinsic and extrinsic motivation. Psychological Inquiry, 11(4), 312-318.

Williams, G. C., Grow, V. M., Freedman, Z. R., Ryan, R. M. \& Deci, E.L.(1996). Motivationa Predictors of Weight Loss and Weight-Loss Maintenance. Journal of Personality and Social Psychology, 70(1), 115-126.

Williams, G. C., Saizow, R., Ross, L., \& Deci, E. L. (1997). Motivation underlying caree choice for internal medicine and surgery. Social Science and Medicine, 45(11), 1705-1713.

Williams, G. C., \& Deci, E. L. (1996). Internalization of Biopsychosocial Values by Medical Students: A Test of Self-Determination Theory. Journal of Personality and Social Psychology, 70(4), 767-779. 